Supporting Information

\title{
A statistical model for large-scale peptide identification in databases from tandem mass spectra using SEQUEST
}

Daniel López-Ferrer ${ }^{\#}$, Salvador Martínez-Bartolomé ${ }^{\#}$, Margarita Villar", Mónica Campillos $^{\#, \$}$, Fernando Martín-Maroto ${ }^{\&}$ and Jesús Vázquez ${ }^{\#, *}$

\#Centro de Biología Molecular "Severo Ochoa"-CSIC, 28049 Cantoblanco, Madrid, Spain, and ${ }^{\circledR}$ ThermoFinnigan, River Oaks Parkway 355, San Jose, CA, USA

\section{Table of Contents:}

\section{Results}

Effect of the fraction of correct peptide sequences on the False Discovery Rate estimation

\section{Figures}

Figure S-1. Modeling of data from Table 2 by two gaussian distributions

Figure S-2. Effect of the fraction of correct peptide sequences on the estimation of the False Discovery Rate

Figure S-3. Comparison of the results obtained using our method with those obtained using the method of Keller et al.

\section{Tables}

Table S-1. Parameters of the distribution of false positives for equine.fasta database for different precursor mass windows

Table S-2. Parameters of the distribution of false positives for different protein databases

Table S-3. List of proteins identified in the nuclear proteome of Jurkat cells 
Results

Effect of the fraction of correct peptide sequences on the False Discovery Rate estimation. To analyze the validity of our approximation to estimate the False Positive Ratio at a given $p$-threshold $\left(F D R_{p}\right.$, see Experimental Section), we modeled the distributions of true and false peptide fragmentations using two gaussian distributions, in order to generate the results presented in Table 2. As shown in Fig. S-1, assuming that the fraction of correct peptide sequences was $20 \%$, this model gave a good prediction of these results, and was therefore used to simulate the effect of the fraction of correct peptide sequences.

The fraction of correct peptide sequences was then varied from $10 \%$ to $50 \%$, and the exact value of $F D R_{p}$ as a function of $p$ was calculated in each case (Fig. S-2, lines), using the distributions calculated above. $F D R_{p}$ was also calculated from the observed and expected number of spectra employing the approximation described in the Experimental Section (Fig. S-2, squares and circles). As shown in Fig. S-2, this approximation was very good even when the fraction of correct peptide sequences was as high as $50 \%$, thus supporting the validity of our method for the calculation of $F D R_{p}$. 
Figures and Figure Legends

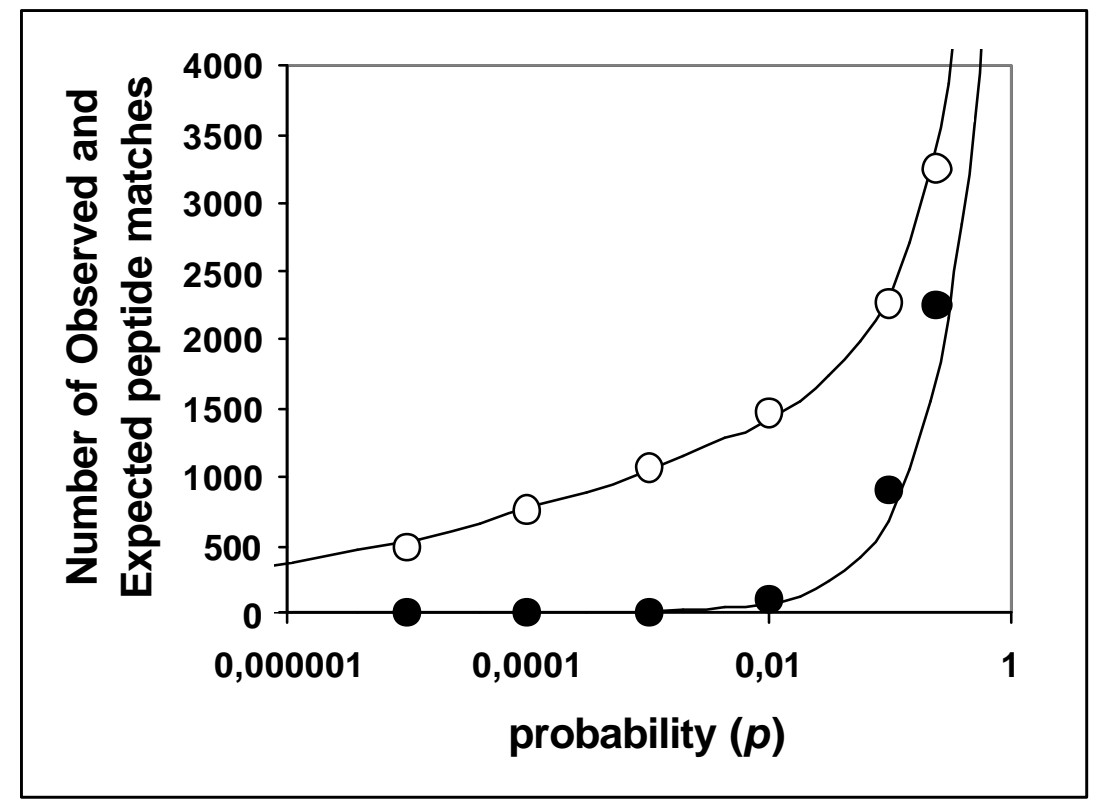

Fig. S-1.- Modeling of data from Table 2 by two gaussian distributions. Points represent the number of observed (empty circles) and expected peptide matches (filled circles), taken from Table 2. The lines are the theoretical predictions of these values according to the gaussian distributions, assuming that the fraction of correct peptide matches was $20 \%$. 


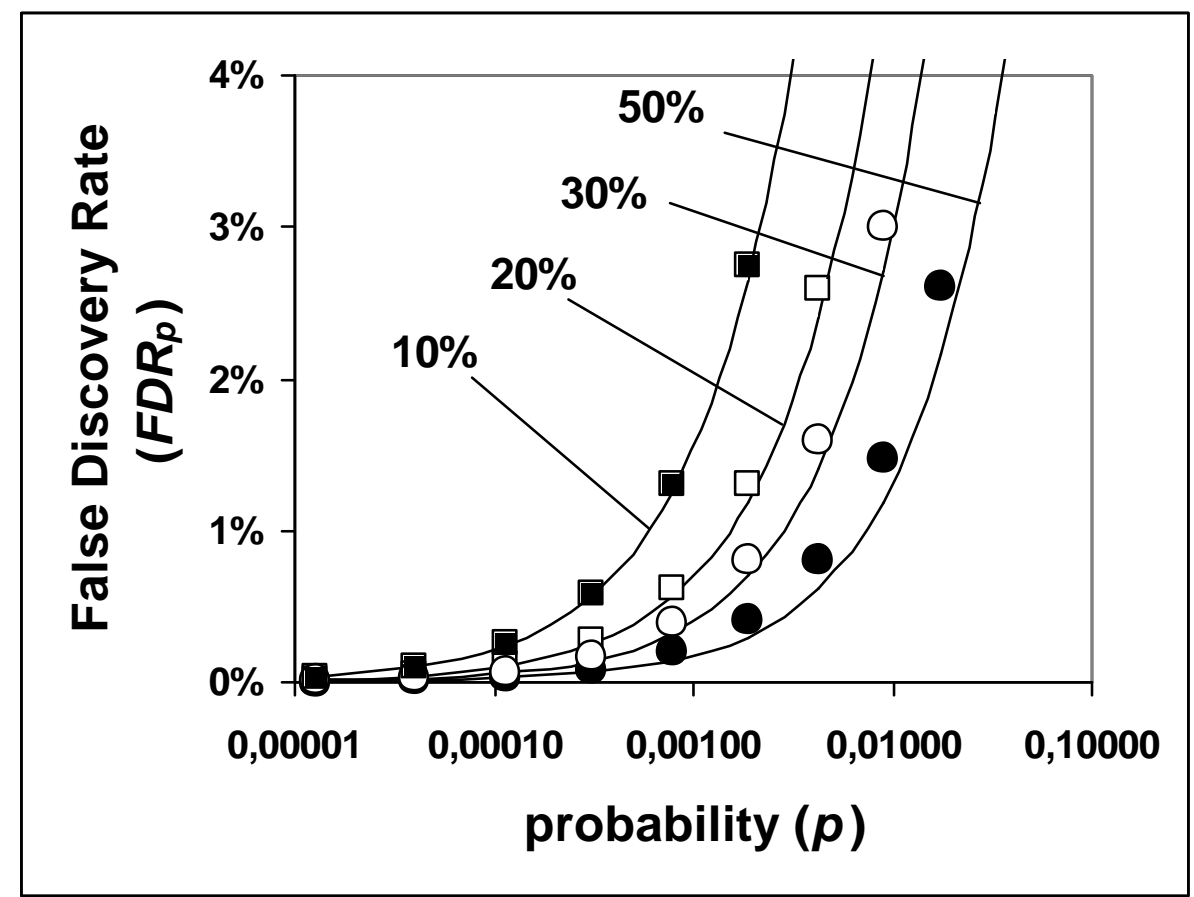

Fig. S-2.- Effect of the fraction of correct peptide sequences on the estimation of the False Discovery Rate. The fraction of correct sequences was varied from $10 \%$ to $50 \%$, and $F D R_{p}$ was calculated from the observed and expected number of matches at different $p$-thresholds using the method described in the Experimental Section (squares and circles). The lines represent the simulated exact false positive rate in each case. 


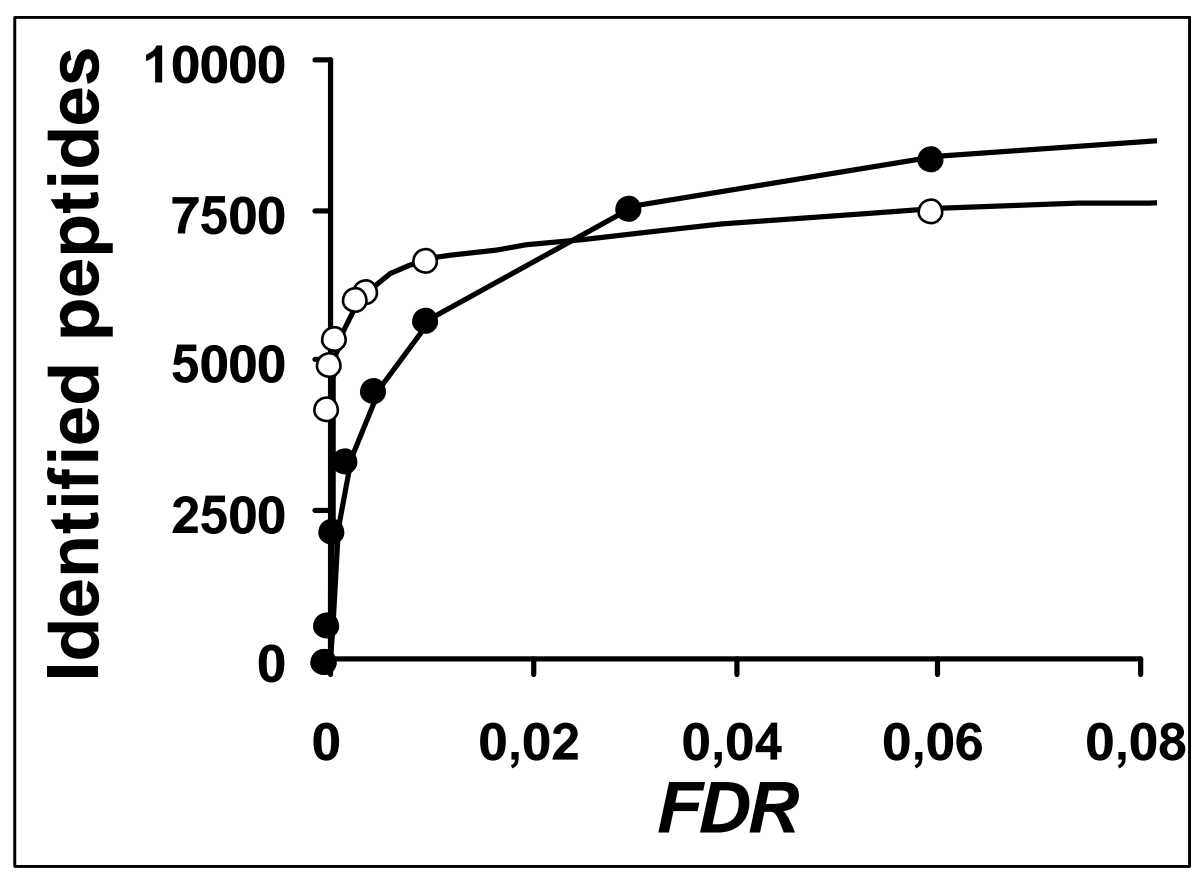

Fig. S-3.- Comparison of the results obtained using our method (empty circles) with those obtained using the method of Keller et al. ${ }^{4}$ (PeptideProphet) (filled circles) for the analysis of a proteome of cells in culture. The starting amount of protein was $240 \mu \mathrm{g}$, and the total number of spectra analyzed was 156.742 . 
Tables

Table S-1

Parameters of the distribution of false positives for the equine.fasta database using different precursor mass windows

\begin{tabular}{|c|c|c|c|c|c|c|}
\hline \multirow{2}{*}{$\begin{array}{c}\text { Precursor mass } \\
\text { window (Da) }\end{array}$} & \multicolumn{2}{|c|}{ charge 2+ } & \multicolumn{2}{|c|}{ charge 3+ } & \multirow[b]{2}{*}{$\mu_{D}$} & \multirow[b]{2}{*}{$\sigma_{D}$} \\
\hline & $\mu_{x}$ & $\sigma_{x}$ & $\mu_{x}$ & $\sigma_{x}$ & & \\
\hline 1 & $-0,60$ & $\overline{0,45}$ & $-0,31$ & $\overline{0,41}$ & 0,48 & 0,22 \\
\hline 2 & $-0,44$ & 0,37 & $-0,15$ & 0,35 & 0,42 & 0,19 \\
\hline 4 & $-0,31$ & 0,32 & $-0,02$ & 0,31 & 0,37 & 0,18 \\
\hline 6 & $-0,25$ & 0,30 & 0,04 & 0,29 & 0,35 & 0,17 \\
\hline 8 & $-0,20$ & 0,29 & 0,08 & 0,28 & 0,34 & 0,17 \\
\hline
\end{tabular}

\section{Table S-2}

Parameters of the distribution of false positives for different protein databases

\begin{tabular}{|c|c|c|c|c|c|c|}
\hline \multirow[b]{2}{*}{ Database } & \multicolumn{2}{|c|}{ charge 2+ } & \multicolumn{2}{|c|}{ charge 3+ } & \multirow[b]{2}{*}{$\mu_{D}$} & \multirow[b]{2}{*}{$\sigma_{D}$} \\
\hline & $\mu_{x}$ & $\sigma_{x}$ & $\mu_{x}$ & $\sigma_{x}$ & & \\
\hline nr.fasta & 0,34 & 0,26 & 0,61 & 0,25 & 0,13 & 0,24 \\
\hline swissprot.fasta & 0,25 & 0,26 & 0,52 & 0,25 & 0,24 & 0,12 \\
\hline human.fasta & 0,21 & 0,26 & 0,48 & 0,25 & 0,24 & 0,12 \\
\hline rat.fasta & 0,19 & 0,26 & 0,46 & 0,25 & 0,24 & 0,13 \\
\hline yeast.fasta & 0,07 & 0,26 & 0,34 & 0,26 & 0,27 & 0,14 \\
\hline equine.fasta & $-0,44$ & 0,37 & $-0,14$ & 0,35 & 0,41 & 0,19 \\
\hline
\end{tabular}


Table S-3

\section{List of proteins identified in the nuclear proteome of Jurkat cells}

\section{PROTEINS IDENTIFIED FROM PEPTIDES WITH $p<10^{-5}$}

gi|123678|sp|P 07900|HS9A_HUMAN Heat shock protein HSP 90-alpha (HSP 86)gi|32488|emb|CAA33259.1| un gi|26454828|gb|AAH40844.1| NSAP1 protein [Homo sapiens] gi|442618|pdb|1ADS| Aldose Reductase (E.C.1.1.1.21) Complex With Nadphgi|576362|pdb|2ACQ| Aldose gi|4505773|ref|NP_002625.1| prohibitin [Homo sapiens]gi|464371|sp|P35232|PHB_HUMAN Prohibitingi|2 gi|72222|pir||HHHU84 heat shock protein 90 -beta [validated] - humangi|306891|gb|AAA36025.1| 90kDa gi|4503545|ref|NP_001961.1| eukaryotic translation initiation factor 5A; eIF5AI [Homo sapiens] gi|2 gi| 19744759|gb|AALL6658.1| guanine nucleotide exchange factor GEF-H1 [Homo sapiens] gi|21594340|gb|AAH31828.1| Kinesin heavy chain member 2 [Homo sapiens]

gi 29436380 gb|AAH49849.1| Similar to myosin, heavy polypeptide 9, non -muscle [Homo sapiens] gi|31565122|gb|AAH53521.1| SPTAN1 protein [Homo sapiens] gi|33875771|gb|AAH00712.2| KIFC1 protein [Homo sapiens] gi|5030431|gb|AAA61281.2| vimentin [Homo sapiens] gi|14327942|gb|AAH09195.1| TRA1 protein [Homo sapiens] gi| 15680282|gb|AAH14504.1| P4HB protein [Homo sapiens] gi|15010550|gb|AAK74072.1| heat shock protein gp96 precursor [Homo sapiens] gi|21361456|ref|NP_055425.2 | alpha glucosidase II alpha subunit; likely ortholog of mouse G2an alph gi| $17318569 \mid$ ref|NP_006112.2| keratin 1; Keratin-1; cytokeratin 1; hair alpha protein [Homo sapiens] gi|20127488|ref|NP_005889.2| membrane component, chromosome 11, surface marker 1 [Homo sapiens]gi| gi|1070482|pir||ROHU thiosulfate sulfurtransferase (EC 2.8.1.1) - humangi|432376|emb|CAA42060.1| r gi|1362844|pir||S57499 isocitrate dehydrogenase (NADP) (EC 1.1.1.42) precursor, mitochondrial - hum gi|14326411|gb|AAK60260.1| short heat shock protein 60 Hsp60s 1 [Homo sapiens] gi|19923315|ref|NP_005403.2| serine hydroxymethyltransferase 2 (mitochondrial) [Homo sapiens] gi|62 gi|20070384|ref|NP_612642.1| hypothetical protein MGC5352 [Homo sapiens]gi|14198272|gb|AAH08196.1] gi|21618939|gb|AAH32008.1| Mitochondrial ribosomal protein L50 [Homo sapiens]

gi|21735621|ref|NP_005909.2| mitochondrial malate dehydrogenase precursor [Homo sapiens] gi|30584131|gb|AAP36314.1| Homo sapiens cytochrome c, somatic [synthetic construct] gi|30585019|gb|AAP36782.1| Homo sapiens ubiquinol-cytochrome $\mathrm{c}$ reductase hinge protein [synthetic $\mathrm{c}$ gi|30585339|gb|AAP36942.1| Homo sapiens ATP synthase, $\mathrm{H}+$ transporting, mitochondrial F1 complex, al gi|31542947|ref|NP_002147.2| heat shock 60kDa protein 1 (chaperonin); mitochondrial matrix protein gi|4028622|gb|AAC96332.1| chaperonin 10-related protein [Homo sapiens]

gi|4758076|ref|NP_004068.1| citrate synthase precursor; Citrate synthase, mitochondrial [Homo sapie gi|4930073|pdb|1P32|A Chain A, Crystal Structure Of Human P32, A Doughnut-Shaped Acidic Mitochondri gi|11360295|pir||T50630 hypothetical protein DKFZp762N0610.1 - human (fragment)gi|8655683|emb|CAB9 gi|11528469|gb|AAG37291.1| humanized L1/ubiqutin hybrid protein [synthetic construct] gi|12053297|emb|CAB66834.1| hypothetical protein [Homo sapiens]

gi|13436308|gb|AAH04945.1| Unknown (protein for IMAGE:3615335) [Homo sapiens] gi|13938297|gb|AAH07276.1| HSPA8 protein [Homo sapiens]

gi|19263762|gb|AAH25362.1| Unknown (protein for IMAGE:5013430) [Homo sapiens] gi|21281677|ref|NP_644810.1| T-cell activation WD repeat protein [Homo sapiens]gi|21205868|gb|AAM4 gi|27734703|ref|NP 775882.1| hypothetical protein FLJ38426 [Homo sapiens] gi|21755073|dbj|BAC04621. gi|34526758|dbj|BAC85280.1| unnamed protein product [Homo sapiens] gi|34532000|dbj|BAC86287.1| unnamed protein product [Homo sapiens] gi|37542862|ref|XP_350876.1| similar to hypothetical protein [Homo sapiens] gi|37543966|ref|XP_090256.5| similar to Heat shock cognate $71 \mathrm{kDa}$ protein [Homo sapiens] gi|4096748|gb|AAC99998.1| ORF; Method: conceptual translation supplied by author. [Homo sapiens] gi|4204880|gb|AAD11466.1| heat shock protein [Homo sapiens]

gi|8923503|ref|NP_060337.1| hypothetical protein FLJ20534 [Homo sapiens]gi|7020706|dbj|BAA9 1241.1| gi|10121863|gb|AAG13404.1| topoisomerase II alpha-3 [Homo sapiens]

gi|105295|pir||C40040 alternative splicing factor ASF-3 - human

gi|1082363|pir||A56619 female sterile homeotic (fsh) homolog RING3 - humangi|182769|gb|AAA68890.1| gi|10863945|ref|NP_066964.1| ATP-dependant DNA helicase II; X-ray repair complementing defective re gi|12083875|gb|AAG 48933.1 | polybromo -1 [Homo sapiens]

gi|1213639|gb|AAC18697.1| scaffold attachment factor B [Homo sapiens]

gi|13016655|emb|CAC29371.1| dJ64K7.3.2 (heterogenous nuclear ribonucleoprotein RALY or autoantigen gi 130781|sp|P09874|PPOL_HUMAN Poly [ADP-ribose] polymerase-1 (PARP-1) (ADPRT) (NAD(+) ADP-ribosylt gi|13124667|sp|O43143|DD15_HUMAN Putative pre-mRNA splicing factor RNA helicase (DEAH box protein 1 gi|13124883|ref|NP_036443.1| HsKin17 protein [Homo sapiens]gi|3850704|emb|CAA06462.1| Kin17 [Homo gi| 13325421|gb|AAH04511.1| Similar to heterogeneous nuclear ribonucleoprotein H3 (2H9) [Homo sapien gi| 13384620 |ref|NP_079555.1| heterogeneous nuclear ribonucleoprotein K [Mus musculus]gi|14165435|r gi|1362789|pir||A57099 DNA -activated protein kinase, catalytic subunit - human

gi|13629286|ref|XP_001541.2| heterogeneous nuclear ribonucleoprotein R [Homo sapiens] gi|13775200|ref|NP_112577.1| SF3b10 [Homo sapiens]gi|20140757|sp|Q9BWJ5|S3B5_HUMAN Splicing factor gi| $13879574|\mathrm{gb}|$ AAH06769.1| NOLC1 protein [Homo sapiens] gi| $13937888|\mathrm{gb}|$ AAH07052.1| HNRPC protein [Homo sapiens] gi|14705264|gb|AAH00903.2| HMGB2 protein [Homo sapiens] gi|14733321|ref|XP_041221.1| similar to RNA-binding protein S1, serine-rich domain; SR protein [Hom gi| 14750549 |ref|XP_029045.1| similar to KIAA0560 protein [Homo sapiens]

gi|15422161|gb|AAK95854.1| transcriptional co-repressor Sin3A [Homo sapiens]

gi|1549243|gb|AAC50695.1| SWI/SNF complex $60 \mathrm{KDa}$ subunit [Homo sapiens]

gi|15593004|gb|AAL02174.1| poly(ADP-ribose) polymerase [Homo sapiens]

gi| 16501181|emb|CAD10078.1| SI:dZ150F13.2 (novel protein similar to human splicing factor 3a, subun gi|16876910|gb|AAH16736.1| HNRPF protein [Homo sapiens]
SUBCELLULAR LOCATION

cytoplasm

cytoplasm

cytoplasm

cytoplasm

cytoplasm

cytoplasm

cytoskeleton

cytoskeleton

cytoskeleton

cytoskeleton

cytoskeleton

cytoskeleton

endoplasmic reticulum

endoplasmic reticulum

endoplasmic reticulum

endoplasmic reticulum

keratin

membrane

mitochondrion

mitochondrion

mitochondrion

mitochondrion

mitochondrion

mitochondrion

mitochondrion

mitochondrion

mitochondrion

mitochondrion

mitochondrion

mitochondrion

mitochondrion

mitochondrion

non assigned

non assigned

non assigned

non assigned

non assigned

non assigned

non assigned

non assigned

non assigned

non assigned

non assigned

non assigned

non assigned

non assigned

non assigned

nucleus

nucleus

nucleus

nucleus

nucleus

nucleus

nucleus

nucleus

nucleus

nucleus

nucleus

nucleus

nucleus

nucleus

nucleus

nucleus

nucleus

nucleus

nucleus

nucleus

nucleus

nucleus

nucleus

nucleus

nucleus 
gi|1705520|sp|P33991|MCM4_HUMAN DNA replication licensing factor MCM4 (CDC21 homolog) (P1-CDC21)gi gi|1732421|gb|AAB51325.1| C2f [Homo sapiens] gi|14602666|gb|AAH09856.1|C2f protein [Homo sapiens] gi|17390815|gb|AAH18349.1|ZNFN1A1 protein [Homo sapiens]gi|328800 15|gb|AAP88838.1| zinc finger pr gi|18032214|gb|AAL56648.1| histone acetyltransferase MYST1 [Homo sapiens] gi| 18203334|sp|Q9P2I0|CPSB_HUMAN Cleavage and polyadenylation specificity factor, $100 \mathrm{kDa}$ subunit ( gi|19111164|ref|NP_579932.1] SMT3 (supressor of mif two, 3) homolog 2 [Mus musculus]gi|19424298|re gi| 19913406 ref|NP_001058.2 DNA topoisomerase II, alpha isozyme; topoisomerase (DNA) II alpha (170 gi|19923217|ref|NP_002408.2 antigen identified by monoclonal antibody Ki-67; Proliferation-related gi|19924129|ref|NP_005723.2| RAD50 homolog isoform 1 [Homo sapiens]gi|1518806|gb|AAB07119.1| Rad50 gi|20454879|sp|Q13769|CV19_HUMAN Protein C22orf19 (NF2/meningioma region protein pK1.3) (Placental gi|20521660|dbj|BAA34508.2| KIAA0788 protein [Homo sapiens] gi|20809640|gb|AAH28936.1| ZNFN1A2 protein [Homo sapiens] gi|21104432|dbj|BAB93486.1| member RAS oncogene family [Homo sapiens] gi|21237802|ref|NP_003065.2| SWI/SNF-related matrix-associated actin-dependent regulator of chromat gi|21327715|ref|NP 006697.2 | transcription elongation regulator 1; transcription factor CA150; TATA gi|21626466|ref|NP_061322.2| matrin 3 [Homo sapiens]gi|12643409|sp|P43243|MAT3_HUMAN Matrin 3gi|3 gi|21758324|dbj|BAC05286.1| unnamed protein product [Homo sapiens] gi|27552794|gb|AAH41361.1| KIAA0 gi|22085790|gb|AAM90580.1| cytidine monophospho-sialic acid synthase [Homo sapiens] gi|22208967|ref|NP_665906.1 high mobility group AT-hook 1 isoform a; nonhistone chromosomal high-m gi|22531287|gb|AAM96005.1|interferon-inducible IFI 16 [Homo sapiens]

gi|2326524|emb|CAA55967.1| IFI-4 [Homo sapiens]gi|12711292|emb|CAA67169.1| dsRNA adenosine deamina gi|24415404|ref|NP_055426.1| MDN1, midasin homolog [Homo sapiens]gi|24212017|sp|Q9NU22|MDN1_HUMAN gi|24987336|pdb|1H2V|C Chain C, Structure Of The Human Nuclear Cap-Binding-Complex (Cbc) gi $|2554638|$ pdb $\mid 2$ U1A| Rna Binding Domain 2 Of Human U1a Protein, Nmr, 20 Structures gi|2664250|emb|CAA34500.1| unnamed protein product [Homo sapiens]

gi|284156|pir||S22765 heterogeneous ribonuclear particle protein U - humangi|32358|emb|CAA46472.1| gi|285999|dbj|BAA02805.1| KIAA0017 [Homo sapiens]

gi|288567|emb|CAA78815.1| DNA topoisomerase II [Homo sapiens]

gi|30411083|gb|AAH52302.1| THOC4 protein [Homo sapiens]

gi|31455238|gb|AAH00506.3| HNRPA2B1 protein [Homo sapiens]

gi|32879913|gb|AAP88787.1| EBNA-2 co-activator (100kD) [Homo sapiens]

gi|32959908|emb|CAE1 1897.1| small nuclear ribonucleoprotein Sm D1 [Homo sapiens]

gi|33469968|ref|NP 005907.3| minichromosome maintenance protein 7 isoform 1; DNA replication licens

gi|33873840|gb|AAH14046.1| SUPT16H protein [Homo sapiens]gi|33875582|gb|AAH00565.1| SUPT16H protei

gi|33874022|gb|AAH08895.2| HNRPM protein [Homo sapiens]

gi|33879558|gb|AAH27708.1| SFPQ protein [Homo sapiens]

gi|33942072|ref|NP_006577.2 | trinucleotide repeat containing 5; CAG repeat containing; expanded rep

gi|34740329|ref|NP 919223.1| heterogeneous nuclear ribonucleoprotein A3 [Homo sapiens] gi|37549323 |

gi|34783967|gb|AAH56894.1| Unknown (protein for IMAGE:6012257) [Homo sapiens]

gi|37078490|sp|Q96AE4|FUB1_HUMAN Far upstream element binding protein 1 (FUSE binding protein 1) (F gi|37078499|sp|Q96I24|FUB3_HUMAN Far upstream element binding protein 3 (FUSE binding protein 3) gi|37231|emb|CAA48197.1| DNA topoisomerase II [Homo sapiens]

gi|37539051|ref|XP_353622.1| similar to general transcription factor II, i isoform 1; BTK -associate

gi|37541487|ref|XP_015755.4| similar to Heterogeneous nuclear ribonucleoprotein A1 (Helix-destabili gi|37545051|ref|XP_113962.2| similar to KIAA0650 protein [Homo sapiens]

gi|37546338|ref|XP_351931.1| similar to ATP-dependent DNA helicase II, $70 \mathrm{kDa}$ subunit (Lupus Ku aut gi|37550782 |ref|XP 350800.1 | similar to ATP-dependent DNA helicase II, 70 kDa subunit (Lupus Ku aut gi|37556028|ref|XP_351787.1| similar to Heterogeneous nuclear ribonucleoprotein A1 (Helix-destabili gi|420052|pir||A44192 heterogeneous nuclear ribonucleoprotein C-like protein - human gi|4503297|ref|NP_001348.1| DEAH (Asp-Glu-Ala-His) box polypeptide 9 isoform 1; DEAD/H (Asp-Glu-Ala gi|4503351|ref|NP 001370.1| DNA (cytosine-5-)-methyltransferase 1; DNA methyltransferase; DNA methy gi|4503551|ref|NP_001410.1| ELAV (embryonic lethal, abnormal vision, Drosophila)-like 1 (Hu antigen gi|4507857|ref|NP_003461.1| ubiquitin specific protease 7 (herpes virus-associated); Herpes virus-a gi|4507903|ref|NP_003375.1| vaccinia related kinase 1; Vaccinia-related kinase-1 [Homo sapiens]gi| gi|4557453|ref|NP_001264.1| chromodomain helicase DNA binding protein 4; Mi-2b [Homo sapiens]gi|59 gi| 4557645|ref|NP_001524.1| heterogeneous nuclear ribonucleoprotein L [Homo sapiens]gi|133274|sp|P gi|4753161|ref|NP_001511.1| general transcription factor IIIC, polypeptide 1, alpha 220kDa; general gi|4757948|ref|NP_004652.1| cell division cycle protein 23; anaphase-promoting complex subunit 8 [H gi|4758112|ref|NP_004631.1| HLA-B associated transcript 1; HLA-B associated transcript-1; DEAD-box gi|4758138|ref|NP_004387.1| DEAD (Asp-Glu-Ala-Asp) box polypeptide 5; DEAD box-5 [Homo sapiens]gi| gi|4758404|ref|NP_004468.1| FSHD region gene 1; Facioscapulohumeral muscular dystrophy region genegi|4758542|ref|NP_004490.1| heterogeneous nuclear ribonucleoprotein AB isoform b; hnRNP type A/B pr gi|4759158|ref|NP_004588.1| small nuclear ribonucleoprotein polypeptide D2; snRNP core protein D2 [ gi|4826686|ref|NP_004930.1| DEAD (Asp-Glu-Ala-Asp) box polypeptide 1; DEAD box-1 [Homo sapiens]gi] gi $4827040 \mid$ ref|NP_005110.1 thyroid hormone receptor-associated protein, $150 \mathrm{kDa}$ subunit [Homo sapi gi|4885377|ref|NP_005311.1| H1 histone fa mily, member 3; histone H1c [Homo sapiens]gi|121925|sp|P1 gi|4885381|ref|NP_005313.1| H1 histone family, member 5 [Homo sapiens] gi|19856407|sp|P16401|H15_HU gi|4885399|ref|NP_005436.1| chondroitin sulfate proteoglycan 6 (bamacan); human chromosome-associat gi| 4885421|ref|NP_005333.1| high-mobility group box 3; high-mobility group (nonhistone chromosomal) gi|4885423|ref|NP_005454.1| heterogeneous nuclear ribonucleoprotein D-like; A+U -rich element RNA bi gi|4995271|emb|CAB43980.1| dJ1J6.1 (topoisomerase (DNA) I) [Homo sapiens] gi|5031753|ref|NP_005511.1| heterogeneous nuclear ribonucleoprotein H1 [Homo sapiens]gi|1710632|sp gi|505104|dbj|BAA06686.1| KIAA0071 [Homo sapiens]

gi|5453840|ref|NP 006377.1| DEAD (Asp-Glu-Ala-Asp) box polypeptide 17 isoform 1; probable RNA-depen gi|5453994|ref|NP_006256.1|RAD21 homolog; protein involved in DNA double-strand break repair; nucl gi|5454172|ref|NP_006288.1|X-ray repair cross complementing protein 1; X-ray-repair, complementing gi|5533375|gb|AAD45156.1| cell division control protein 16 [Homo sapiens] gi|5542014|emb|CAB51168.1| dyskerin [Homo sapiens] gi|5764101|gb|AAD51135.1| polynucleotide kinase-3'-phosphatase [Homo sapiens] gi|5803036|ref|NP_006796.1| heterogeneous nuclear ribonucleoprotein A0; hnRNA binding protein [Homo gi|5803167|ref|NP_006793.1| splicing factor 3a, subunit 3, 60kDa; pre-mRNA splicing factor SF3a (60 gi|5870129|gb|AAD54488.1| small nuclear ribonucleoprotein B [Homo sapiens] gi 5870276 $\mathrm{gb} \mid$ AAD54507.1] MTDM_HUMAN [AA 1 - 966]; DNA METHYLTRANSFERASE; DNA METASE; MCMT; M.HSAI gi|5901928|ref|NP_008938.1| cleavage and polyadenylation specific factor 6, 68 kD subunit; pre-mRNA nucleus gi|5902076|ref|NP_008855.1| splicing factor, arginine/serine-rich 1 (splicing factor 2, alternate s nucleus 
gi|6005747|ref|NP_009143.1| ring finger protein 2 [Homo sapiens] gi|1785643|emb|CAA71596.1| dinG [H gi|6005757|ref|NP_009123.1| chromatin-specific transcription elongation factor large subunit [Homo gi|602021|emb|CAA29961.1| unnamed protein product [Homo sapiens] gi|6708282|gb|AAF25871.1| lens epithelium-derived growth factor p52 [Homo sapiens] gi|6980832|pdb|1DEW|A Chain A, Crystal Structure Of Human Ape1 Bound To Abasic Dnagi|6980833|pdb|1 gi|7387740|sp|Q93080|H2BL_HUMAN Histone H2B.1 (H2B/1)gi|1568557|emb|CAB02545.1| histone H2B [Homo gi|7427519|ref|NP_005906.2| minichromosome maintenance protein 6; DNA replication licensing factor gi|7446345|pir||G01211 $54 \mathrm{kDa}$ protein - humangi|407308|gb|AAA03427.1| 54 kDa proteingi|2808511|em gi|7512403|pir||T13159 E1B-55kDa-associated protein - humangi|3319956|emb|CAA07548.1|E1B-55kDa-as gi|7706254|ref|NP_057018.1| nucleolar protein NOP5/NOP58 [Homo sapiens] gi|17380155|sp|Q9Y2X3|NOP5_ gi|870747|dbj|BAA09524.1| heterogeneous nuclear ribonucleoprotein D (hnRNP D) [Homo sapiens] gi|9624998|ref|NP_062543.1| heterogeneous nuclear ribonucleoprotein $\mathrm{H2}$; heterogeneous nuclear ribon gi|9714272|emb|CAC01407.1| double stranded RNA binding nuclear protein, ILF3 [Homo sapiens] gi|9954649|gb|AAG09061.1|XRCC1 DNA repair protein [Homo sapiens]

gi|9956070|gb|AAG02007.1| similar to Homo sapiens mRNA for KIAA0723 protein with GenBank Accession gi|30583783|gb|AAP36140.1| Homo sapiens histone deacetylase 1 [synthetic construct]

construction)

gi|30583881|gb|AAP36189.1| Homo sapiens fibrillarin [synthetic construct] construction)

gi|30584163|gb|AAP36330.1| Homo sapiens high-mobility group box 1 [synthetic construct]

construction)

gi|30584409|gb|AAP36457.1| Homo sapiens RuvB-like 1 (E. coli) [synthetic construct]

construction)

gi|30584915|gb|AAP36714.1| Homo sapiens zinc finger protein 24 (KOX 17) [synthetic construct]

construction)

gi|30585117|gb|AAP36831.1| Homo sapiens c-myc binding protein [synthetic construct]

construction)

gi|33873325|gb|AAH10132.1| KHDRBS1 protein [Homo sapiens]

gi|4503841|ref|NP 001460.1| thyroid autoantigen 70kDa (Ku antigen); thyroid autoantigen $70 \mathrm{kD}$ (Ku an

gi|4504771|ref|NP_002203.1| integrin beta 4 binding protein isoform a; eukaryotic translation initi

gi|4757900|ref|NP_004334.1| calreticulin precursor; Sicca syndrome antigen A (autoantigen Ro; calre reticulum

gi|15192188|gb|AAK64620.1| protease [Human immunodeficiency virus type 1]

gi|27802720|emb|CAD60810.1| SI:bZ1O18.2 (novel protein similar to human THO complex 2 (THOC2)) [Dan

gi|14250148|gb|AAH08492.1| Ribosomal protein L3 [Homo sapiens]

gi|36142|emb|CAA44568.1| ribosomal protein homologous to yeast S24 [Homo sapiens]

gi|4506631|ref|NP_000980.1| ribosomal protein L30; 60S ribosomal protein L30 [Homo sapiens]gi|6677

gi|9966867|ref|NP 065123.1 eIF-5A2 protein [Homo sapiens] gi|20864843|ref|XP 130812.1| similar to

gi|4503471|ref|NP_001393.1| eukaryotic translation elongation factor 1 alpha 1; CTCL tumor antigen;

nucleus

nucleus

nucleus

nucleus

nucleus

nucleus

nucleus

nucleus

nucleus

nucleus

nucleus

nucleus

nucleus

nucleus

nucleus

nucleus

(synthetic

nucleus

(synthetic

nucleus

(synthetic

nucleus

(synthetic

nucleus

(synthetic

nucleus

(synthetic

nucleus / memb rane

nucleus / membrane

nucleus / membrane

nucleus/ endoplasmic

others

others

ribosomes

ribosomes

ribosomes

ribosomes

ribosomes / cytoplasm

\section{PROTEINS IDENTIFIED FROM PEPTIDES WITH $10^{-5}<p<10^{-4}$ \\ (not included in previous table)}

gi|2119276|pir||I38369 beta-tubulin - human (fragment)gi|897763|emb|CAA23844.1| unnamed protein pr gi|28336|emb|CAA45026.1| mutant beta-actin (beta'-actin) [Homo sapiens]

gi|30315658|ref|NP_842565.1| spectrin, beta, non-erythrocytic 1 isoform 2; Spectrin, beta, noneryth gi|27884151|emb|CAD61270.1| SI:zC214P16.4 (novel protein similar to human protein phosphatase 1, ca gi|5031973|ref|NP 005733.1| protein disulfide isomerase-related protein [Homo sapiens] gi|2501205|s gi|20070125|ref|NP_000909.2| prolyl 4-hydroxylase, beta subunit; v-erb-a avian erythroblastic leuke gi|20141424|sp|Q16836|HCDH_HUMAN Short chain 3 -hydroxyacyl-CoA dehydrogenase, mitochondrial precurs gi|28178832|ref|NP_002159.2| isocitrate dehydrogenase 2 (NADP+), mitochondrial precursor; isocitrat gi|14250063|gb|AAH08435.1| Peroxiredoxin 3, isoform a precursor [Homo sapiens]

gi 12230015 |sp|O43169|CYM5 HUMAN Cytochrome b5 outer mitochondrial membrane isoform precursorgi|26 gi|4557525|ref|NP_000099.1| dihydrolipoamide dehydrogenase precursor; E3 component of pyruvate dehy gi|11139093|gb|AAG31605.1| GrpE-like protein cochaperone [Homo sapiens] gi|1071813|pir||A53719 glutamate dehydrogenase [NAD(P)] (EC 1.4.1.3) 2 precursor - humangi|31815|e gi|30268206|emb|CAD89914.1| hypothetical protein [Homo sapiens]

gi|7245516|pdb|1CMI|A Chain A, Structure Of The Human PinLC8 DIMER WITH A BOUND PEPTIDEgi|7245517| gi|23241743|gb|AAH35409.1| Transducin beta-like 3 [Homo sapiens]

gi|7512911|pir||T08769 hypothetical protein DKFZp586K011.1 - human (fragment)gi|4884343|emb|CAB432

gi|10437063 dbj|BAB14971.1| unnamed protein product [Homo sapiens]

gi| 11544739 emb|CAC17645.1| dJ776P7.2 (WD repeat domain 3) [Homo sapiens]

gi|18606048|gb|AAH22802.1| Unknown (protein for IMAGE:5443781) [Homo sapiens]

gi|33946293|ref|NP_861446.2| hypothetical protein FLJ20403 isoform 2; zinc finger protein 326 [Homo gi|5881961|gb|AAD55138.1| Dhm1 -like protein [Homo sapiens]

gi|4503249|ref|NP 003463.1| DEK oncogene (DNA binding); DEK gene [Homo sapiens] gi|37549944|ref|XP_293903.2| similar to Fibrillarin (Nucleolar protein 1) [Homo sapiens]

gi|13699256|ref|NP_054829.2| protein phosphatase 1 regulatory inhibitor subunit 8 isoform alpha; ar gi|24432016|ref|NP_079087.2| pre-mRNA cleavage factor I, $59 \mathrm{kDa}$ subunit [Homo sapiens] gi|22450806 gi|11320942|gb|AAG33967.1| BRG1-Associated Factor 250a [Homo sapiens] gi|21619414|gb|AAH32128.1| DDX39 protein [Homo sapiens]

gi|37549676|ref|XP_351560.1| similar to MGC37309 protein [Homo sapiens]

gi|4557491|ref|NP_001315.1| cleavage stimulation factor subunit 1 [Homo sapiens] gi|461848|sp|Q0504 gi|20149524|ref|NP_003160.2| suppressor of Ty 5 homolog [Homo sapiens] gi|2065177|emb|CAA73326.1| S gi|21619317|gb|AAH31779.1| Similar to KIAA0052 protein [Homo s apiens]

gi|543010|pir||S41768 splicing factor homolog - humangi|348239|gb|AAC37578.1| [Human mRNA, complet gi|5032169|ref|NP_005643.1| telomeric repeat binding factor 2; telomeric repeat binding protein 2 [ gi|31795540|ref|NP_853556.1| replication factor C 5 isoform 2; RFC, $36.5 \mathrm{kD}$ subunit; activator 136 gi|37541035|ref|XP_290506.2 | similar to Splicing factor 3B subunit 2 (Spliceosome associated protei gi|7661920|ref|NP_055555.1| DEAD (Asp-Glu-Ala-Asp) box polypept ide 48 [Homo sapiens]gi|20532400|sp gi|4507241|ref|NP_003137.1| structure specific recognition protein 1; recombination signal sequence gi|20270337|ref|NP_620147.1| senescence downregulated leo1-like [Homo sapiens] gi|17390326|gb|AAH18 gi|3191965|emb|CAB05110.1| bK286B10.3.1 (minichromosome maintenance deficient (S. cerevisiae) 5 (ce

cytoskeleton

cytoskeleton

cytoskeleton

endoplasmic reticulum

endoplasmic reticulum

endoplasmic reticulum

mitochondrion

mitochondrion

mitochondrion

mitochondrion

mitochondrion

mitochondrion

mitochondrion

non assigned

non assigned

non assigned

non assigned

non assigned

non assigned

non assigned

non assigned

nucleus

nucleus

nucleus

nucleus

nucleus

nucleus

nucleus

nucleus

nucleus

nucleus

nucleus

nucleus

nucleus

nucleus

nucleus

nucleus

nucleus

nucleus

nucleus 
gi|13632817|sp|Q9UQ80|P2G4_HUMANProliferation-associated protein 2G4 (Cell cycle protein p38-2G4 h

gi|14726321|ref|XP_001527.4| polymyositis/scleroderma autoantigen 2 (100kD) [Homo sapiens]gi|89285 gi|7657381|ref|NP_055317.1| nuclear matrix protein NMP200 related to splicing factor PRP19 [Homo sa gi|4827042|ref|NP_005111.1| trinucleotide repeat containing 11 (THR-associated protein, 230kDa subu gi|6912654|ref|NP_036565.1| splicing factor 3b, subunit 1, 155kDa; spliceosome-associated factor 15 gi|29568103|ref|NP_003080.2|U1 small nuclear ribonucleoprotein $70 \mathrm{kDa}$; small nuclear ribonucleopro gi|21749550|dbj|BAC03616.1| unnamed protein product [Homo sapiens]

gi|4506901|ref|NP_003008.1| splicing factor, arginine/serine-rich 3; splicing factor, arginine//ser gi|4566530|gb|AAD23390.1| SWI/SNF-related, matrix-associated, actin-dependent regulator of chromati gi|2506974|sp|Q01196|RUN1_HUMAN Runt-related transcription factor 1 (Core-binding factor, alpha $2 \mathrm{~s}$ gi|4506903|ref|NP_003760.1| splicing factor, arginine/serine-rich 9 [Homo sapiens] gi|3929377|sp|Q1 gi|1232077|dbj|BAA12177.1| huMCM2 [Homo sapiens]

gi|7710126|ref|NP_039269.1| ligase III, DNA, ATP-dependent isoform alpha [Homo sapiens]gi|1706481| gi| $28948850 \mid$ pdb|1 1 HH 3 A Chain A, Human Topoisomerase I Ara-C Complex gi|1279913|gb|AAC50591.1| ENX-1 [Homo sapiens]

gi|6857818|ref|NP_008924.1| nucleophosmin/nucleoplasmin 3; nucleoplasmin-3; nucleophosmin/nucleopla gi|22057930|ref|XP_170536.1| similar to eukaryotic translation initiation factor 2, subunit 3 gamma

gi|37078468|sp|Q92945|FUB2_HUMAN Far upstream element binding protein 2 (FUSE binding protein 2) (K gi|21071058|ref|NP_003592.2 | SWI/SNF-related matrix-associated actin-dependent regulator of chromat gi|20270186|ref|NP_004233.1| high mobility group nucleosomal binding domain 3 isoform HMGN3a; thyro gi|38014635|gb|AAH04534.2| Unknown (protein for IMAGE:3139130) [Homo sapiens] gi|37748329|gb|AAH58896.1| DHX9 protein [Homo sapiens] gi|4504255|ref|NP_002097.1| H2A histone family, member Z; H2AZ histone [Homo sapiens] gi|7949045|re gi|30584739|gb|AAP36622.1| Homo sapiens splicing factor, arginine/serine-rich 7, 35kDa [synthetic constr construction)

gi|5803181|ref|NP_006810.1| stress -induced-phosphoprotein 1 (Hsp70/Hsp90-organizing protein) [Homo gi|9716100|emb|CAC01510.1| dJ999L4.1 (novel protein similar to ribosomal protein L12 (RPL12)) [Homo gi|29789090|ref|NP_061185.1| RCC1 -like [Homo sapiens]gi|27526613|emb|CAD13148.1| RCC1 -like protein gi|13124879|ref|NP_002465.1| smooth muscle myosin heavy chain 11 isoform SM1 [Homo sapiens] gi|1343 gi|625305|pir||A61 231 myosin heavy chain nonmuscle form A - human

gi|32015|emb|CAA30026.1| alpha-tubulin [Homo sapiens] gi|1333692|emb|CAA28453.1| unnamed protein pr gi|22748721|ref|NP_689543.1| chromosome 14 open reading frame 31 [Homo sapiens] gi|18088998|gb|AAH2 gi|2135267|pir||S63994 protein disulfide-isomerase (EC 5.3.4.1) ER60 precursor - human gi| 435476|emb|CAA82315.1| cytokeratin 9 [Homo sapiens]

gi|2982114|pdb|1PBH| Crystal Structure Of Human Recombinant Procathepsin B At 3.2 Angstrom Resolut gi|29841421|gb|AAP06453.1| similar to NM_019693 HLA-B associated transcript 1 in Homo sapiens [Schi gi|37550243|ref|XP_171032.3| similar to Succinate dehydrogenase [ubiquinone] flavoprotein subunit, gi|565169|gb|AAB31203.1| early-pregnancy factor, EPF=chaperonin 10 homolog [human, platelets, Pepti gi|28940|emb|CAA27246.1| unnamed protein product [Homo sapiens] gi|219654|dbj|BAA00016.1|F1 beta s gi|21903482|sp|P22695|UCR2_HUMAN Ubiquinol-cytochrome C reductase complex core protein 2, mitochond gi|28372537|ref|NP_777577.1| hypothetical protein LOC197322 [Homo sapiens]gi|22761682|dbj|BAC11654 gi|10434304|dbj|BAB 14211.1| un named protein product [Homo sapiens] gi|14602481|gb|AAH09746.1| FLJ20 gi|13623693|gb|AAH06474.1| Unknown (protein for IMAGE:2820942) [Homo sapiens] gi|284289|pir||S27954 leucine-rich protein - human

gi|28491496|ref|XP_194468.2| similar to hypothetical protein MGC10084 [Homo sapiens] [Mus musculus] gi|7023391|dbj|BAA91947.1| unnamed protein product [Homo sapiens] gi|8922331|ref|NP_060518.1| hypothetical protein FLJ10292 [Homo sapiens]gi|7022229|dbj|BAA91522.1| gi|30354168|gb|AAH51866.1| RING1 protein [Homo sapiens]gi|33877354|gb|AAH02922.2| RING1 protein [H gi|18088489|gb|AAH20786.1| PLRG1 protein [Homo sapiens]

gi|13111899|gb|AAH03118.1| MGC2655 protein [Homo sapiens] gi|33150820|gb|AAP97288.1| MSIN3A [Homo sapiens]

gi|6966969|emb|CAB72445.1 bromodomain containing protein [Homo sapiens]gi|8452874|gb|AAF75126.1| gi|37550368|ref|XP_027964.2| similar to Heterogeneous nuclear ribonucleoprotein A1 (Helix-destabili gi|8671754|gb|AAF78364.1| DAZ associated protein 1 [Homo sapiens]

gi|13124873|ref|NP_001746.1| core-binding factor, beta subunit isoform 2; polyomavirus enhancer bin gi| $482335 \mid$ pir||A42170 zinc finger protein MAZ - human (fragment)

gi|8922679|ref|NP_060695.1|homolog of C. elegans smu -1; ortholog of rat brain-enriched WD-repeat p gi|16307090|gb|AAH09623.1| NPM1 protein [Homo sapiens]

gi|7661958|ref|NP_055554.1| Bcl-2-associated transcription factor [Homo sapiens] gi|1136390|dbj|BAA gi|37550496|ref|XP_209517.2| similar to heterogeneous nuclear ribonucleoprotein K isoform a; dC-str gi|288565|emb|CAA78821.1| DNA topoisomerase II [Homo sapiens]

gi|4759196|ref|NP_004810.1| symplekin [Homo sapiens]gi|8928372|sp|Q92797|SPK_HUMAN Symplekingi|15 gi|23512328|gb|AAH38447.1| COPA protein [Homo sapiens] gi|16306625|gb|AAH01487.1| TARDBP protein [Homo sapiens] gi|4506587|ref|NP_002938.1| replication protein A3, 14kDa; replication protein A3 (14kD) [Homo sapi gi|16307125|gb|AAH09650.1| KIAA0648 protein [Homo sapiens]

gi|5453792|ref|NP_006161.1| nucleolar protein 1, 120kDa; nucleolar protein 1 (120kD) [Homo sapiens] gi|5031653|ref|NP_005863.1| breast carcinoma amplified sequence 2; spliceosome associated protein, gi|4506435|ref|NP_000312.1| retinoblastoma 1 (including osteosarcoma); Retinoblastoma-1 [Homo sapie gi|13654278|ref|NP_112487.1| hypothetical protein DC50 [Homo sapiens]gi|12005807|gb|AAG44629.1| DC gi|5453820|ref|NP_006176.1| nuclear mitotic apparatus protein 1 [Homo sapiens] gi|284337|pir||A4218 gi|12803125|gb|AAH02366.1| DEAD (Asp-Glu-Ala-Asp) box polypeptide 23 [Homo sapiens] gi|12084561|pdb|1FU1|A Chain A, Crystal Structure Of Human Xrcc4gi|12084562|pdb|1FU1|B Chain B, Cr gi|6180013|gb|AAF05753.1| anaphase-promoting complex subunit 5 [Homo sapiens]

cytoplasm

cytoskeleton

cytoskeleton

cytoskeleton

cytoskeleton

cytoskeleton

endoplasmic reticulum

keratin

lysosomes

membrane

mitochondrion

mitochondrion

mitochondrion

mitochondrion

mitochondrion

non assigned

non assigned

non assigned

non assigned

non assigned

non assigned

nucleus

nucleus
nucleus

nucleus

nucleus

nucleus

nucleus

ucleu

nucleus
nucleus

nucleus

nucleus

nucleus

nucleus

nucleus

nucleus

nucleus

nucleus

nucleus

nucleus

nucleus

nucleus

nucleus

nucleus

nucleus

nucleus

nucleus

nucleus

nucleus 
gi|37550770|ref|XP_290471.3| similar to wings apart-like CG3707-PA [Homo sapiens]

gi|8922789|ref|NP_060752.1| paraspeckle protein 1 [Homo sapiens]gi|7023323|dbj|BAA91924.1| unnamed

gi|37231483|gb|AAH01384.2| USP39 protein [Homo sapiens]

gi|34785168|gb|AAH56912.1| Unknown (protein for IMAGE:4941153) [Homo sapiens]

nucleus

gi|3915658|sp|Q08211|DHX9_HUMAN ATP-dependent RNA helicase A (Nuclear DNA helicase II) (NDH II) (DE

gi|5174449|ref|NP_006017.1| H1 histone family, member X [Homo sapiens]gi|6016184|sp|Q92522|H1X_HUM

nucleus

nucleus

nucleus

nucleus gi|21708041|gb|AAH33817.1| PSIP2 protein [Homo sapiens]

nucleus

nucleus

gi|4758356|ref|NP_004102.1| flap structure-specific endonuclease 1; maturation factor-1; DNase IV [

gi|31563531|ref|NP_006594.3| stromal antigen 2 [Homo sapiens]gi|37515276|gb|AAH01765.2| Stromal an

nucleus

nucleus

gi|7263962|emb|CAB81646.1| bA395L14.12 (novel protein similar to small nuclear ribonucleoprotein po

nucleus

gi|37539731|ref|XP_352555.1| similar to 60S ribosomal protein L36 [Homo sapiens] 